\title{
Some Applications of Ordinary Differential Operator to Certain Multivalent Functions
}

\author{
Müfit Şan, ${ }^{1}$ Hüseyin Irmak, ${ }^{1}$ and Ayhan Şerbetçi ${ }^{2}$ \\ ${ }^{1}$ Department of Mathematics, Faculty of Science, Cankırı Karatekin University, 18100 Çankırı, Turkey \\ ${ }^{2}$ Department of Mathematics, Faculty of Science, Ankara University, 06100 Ankara, Turkey
}

Correspondence should be addressed to Hüseyin Irmak; hisimya@yahoo.com

Received 1 July 2015; Accepted 11 August 2015

Academic Editor: Viliam Makis

Copyright (C) 2015 Müfit Şan et al. This is an open access article distributed under the Creative Commons Attribution License, which permits unrestricted use, distribution, and reproduction in any medium, provided the original work is properly cited.

The aim of this paper is to apply the well-known ordinary differential operator to certain multivalent functions which are analytic in the certain domains of the complex plane and then to determine some criteria concerning analytic and geometric properties of the related complex functions.

\section{Introduction, Notations, and Definitions}

Let $\mathscr{A}(\kappa ; p)$ denote the class of functions $f(z)$ of the following form:

$$
\begin{aligned}
& f(z)=z^{\kappa}+a_{p+1} z^{p+1}+a_{p+2} z^{p+2}+\cdots \\
& \left(a_{k} \in \mathbb{C} ; \kappa \in \mathbb{Z}=\{ \pm 1, \pm 2, \pm 3, \ldots\} ; p \in \mathbb{N}\right. \\
& \left.\quad=\{1,2,3, \ldots\}, \mathbb{Z}^{-}=\mathbb{Z}-\mathbb{N}\right),
\end{aligned}
$$

which are analytic and multivalent in the domain

$$
\Delta= \begin{cases}\mathbb{U}, & \text { when } \kappa \in \mathbb{N} \\ \mathbb{D}, & \text { when } \kappa \in \mathbb{Z}^{-},\end{cases}
$$

where $\mathbb{C}$ is the set of complex numbers. As is known, the domains $\mathbb{U}$ and $\mathbb{D}$ are known as unit open disk and punctured open unit disk, respectively. Also let $\mathscr{M}(p):=\mathscr{A}(-p ; p)$ and $\mathscr{T}(p):=\mathscr{A}(p ; p)$ when $p \in \mathbb{N}$.

By differentiating both sides of the function $f(z)$ in the form (1), $q$-times with respect to complex variable $z$, one can easily derive the following (ordinary) differential operator:

$$
f^{(q)}(z)= \begin{cases}\frac{p !}{(p-q) !} z^{p-q}+\sum_{k=p+1}^{\infty} \frac{k !}{(k-q) !} a_{k} z^{k-q}, & \text { if } f \in \mathscr{T}(p) \\ \frac{(p+q-1) !}{(p-1) !}(-1)^{q} z^{-p-q}+\sum_{k=p+1}^{\infty} \frac{k !}{(k-q) !} a_{k} z^{k-q}, & \text { if } f \in \mathscr{M}(p),\end{cases}
$$

where $p \geq q, p \in \mathbb{N}$, and $q \in \mathbb{N}_{0}:=\mathbb{N} \cup\{0\}$.

In this investigation, by applying the differential operator, defined by (3), to certain analytic functions which are multivalent in $\mathbb{U}$ or meromorphic multivalent in $\mathbb{D}$, several criteria, which also include both analytic and geometric properties of univalent functions (see $[1,2])$, for functions $f(z)$ in the classes $\mathscr{M}(p)$ and $\mathscr{T}(p)$, are then determined. In the literature, by using certain operators, several researchers obtained some results concerning functions belonging to the general class $\mathscr{A}(\kappa ; p)$. In this paper, we also determined many results which include starlikeness, convexity, close-toconvexity, and close-to-starlikeness of analytic functions in 
the second section of this paper. One may refer to some results determined by ordinary differential operator in $[3,4]$, some properties of certain linear operators in [5-7], and also certain results appertaining to multivalent functions and some of their geometric and analytic properties in $[8,9]$ in the references.

For the proofs of the main results, we then need to recall the well-known method which was obtained by Jack [10] (see also [11]) and given by the following lemma.

Lemma 1. Let the function $w(z)$ given by

$$
w(z)=c_{n} z^{n}+c_{n+1} z^{n+1}+c_{c+2} z^{c+2}+\cdots \quad(n \in \mathbb{N})
$$

be analytic in $\mathbb{U}$ with $w(z) \not \equiv 0(z \in \mathbb{U})$.

$$
\text { If }
$$

$$
\begin{aligned}
z_{0} & =r e^{i \theta_{0}}, \\
\left|w\left(z_{0}\right)\right| & =\max _{|z| \leq\left|z_{0}\right|}|w(z)|,
\end{aligned}
$$

then

$$
z_{0} w^{\prime}\left(z_{0}\right)=c w\left(z_{0}\right)
$$

where $c$ is real number and $c \geq n \geq 1$.

\section{The Main Results and Their Applications}

Theorem 2. Let $f(z) \in \mathscr{M}(p)$ and $z \in \mathbb{D}$, and also let the following inequality:

$$
q+\mathfrak{R} e\left(\frac{z f^{(1+q)}(z)}{f^{(q)}(z)}\right)<\frac{1}{2}
$$

be true. Then

$$
\mathfrak{R e}\left\{z^{p+q} f^{(q)}(z)\right\} \begin{cases}>0, & \text { when } q \text { is even } \\ <0, & \text { when } q \text { is odd. }\end{cases}
$$

Proof. Let $f(z) \in \mathscr{M}(p)$. By applying the differential operator, defined in (3), to the function $f(z)$, one easily get that

$$
\begin{aligned}
\frac{z^{p+q} f^{(q)}(z)}{\gamma(p, q)} & =1+\sum_{k=p+1}^{\infty} \frac{\Gamma(k, q)}{\gamma(p, q)} a_{k} z^{k+p} \\
& =1+\sum_{k=p+1}^{\infty} d_{k} z^{k+p} \\
& =1+d_{p+1} z^{2 p+1}+d_{p+2} z^{2 p+2}+\cdots \\
& =1+w(z),
\end{aligned}
$$

where $\gamma(p, q)$ and $\Gamma(k, q)$ are defined by

$$
\begin{aligned}
& \gamma(p, q):=\frac{(p+q-1) !}{(p-1) !}(-1)^{q} \\
& \qquad\left(p \geq q ; p \in \mathbb{N} ; q \in \mathbb{N}_{0}\right), \\
& \Gamma(k, q):=\frac{k !}{(k-q) !} \\
&\left(k=p+1, p+2, \ldots ; p \geq q ; p \in \mathbb{N} ; q \in \mathbb{N}_{0}\right),
\end{aligned}
$$

respectively. It is clear that the defined function $w(z)$ has the form in Lemma 1; that is, it is analytic in $\mathbb{U}$ with $w(z) \not \equiv 0$. Upon differentiating of the identity (9), one easily obtains that

$$
p+\frac{z f^{(1+q)}(z)}{f^{(q)}(z)}+q=\frac{z w^{\prime}(z)}{1+w(z)},
$$

$$
(z \in \mathbb{U} ; w(z) \neq-1) .
$$

Now suppose that there exists a point $z_{0} \in \mathbb{U}$ such that

$$
\max \left\{|w(z)|:|z| \leq\left|z_{0}\right|\right\}=\left|w\left(z_{0}\right)\right|=1,
$$

by applying Lemma 1 ; we then have $z_{0} w^{\prime}\left(z_{0}\right)=c w\left(z_{0}\right)(c \geq$ $n \geq 1)$. Thus, in view of the above equality, it can be calculated that

$$
\begin{aligned}
\mathfrak{R} e\left(p+\frac{z f^{(1+q)}(z)}{f^{(q)}(z)}+\left.q\right|_{z=z_{0}}\right) \\
=\mathfrak{R} e\left(\left.\frac{z w^{\prime}(z)}{1+w(z)}\right|_{z=z_{0}}\right)=\frac{c}{2} \geq \frac{2 p+1}{2}
\end{aligned}
$$

which contradicts (7). Hence, we conclude that $|w(z)|<1$ for all $z \in \mathbb{U}$, and Definition (9) yields the inequality

$$
\left|\frac{z^{p+q} f^{(q)}(z)}{\gamma(p, q)}-1\right|=|w(z)|<1,
$$

which is equivalent to (8). Therefore, this completes the desired proof.

Theorem 3. Let $f(z) \in \mathscr{M}(n), g(z) \in \mathscr{M}(m), q \in \mathbb{N}_{0}$, and, also, the following inequality

$$
\mathfrak{R} e\left(\frac{g^{(q)}(z)}{z g^{(1+q)}(z)}\right)>\alpha, \quad\left(\alpha>0 ; z \in \mathbb{U} ; q \in \mathbb{N}_{0}\right)
$$

be satisfied. If

$$
\left|\frac{f^{(1+q)}(z)}{g^{(1+q)}(z)}\right|<[1+\alpha(m-n)] \cdot \frac{\gamma(n, q)}{\gamma(m, q)},
$$

then

$$
\left|\frac{f^{(1+q)}(z)}{g^{(1+q)}(z)}\right|<\frac{\gamma(n, q)}{\gamma(m, q)}, \quad(m>n ; n, m \in \mathbb{N}) .
$$


Proof. Let the functions $f(z) \in \mathscr{M}(n)$ and $g(z) \in \mathscr{M}(m)$ be in the form

$$
\begin{aligned}
& f(z)=z^{-n}+\sum_{k=p+1}^{\infty} a_{k} z^{k}, \\
& g(z)=z^{-m}+\sum_{k=p+1}^{\infty} b_{k} z^{k},
\end{aligned}
$$

respectively. Then, from related differential operator and definitions of the functions $f(z)$ and $g(z)$, determine

$$
\begin{aligned}
& \frac{\gamma(m, q)}{\gamma(n, q)} \cdot \frac{f^{(q)}(z)}{g^{(q)}(z)} \\
& =\frac{z^{-n-q}+\sum_{k=p+1}^{\infty}(\Gamma(k, q) / \gamma(p, q)) a_{k} z^{k+p}}{z^{-m-q}+\sum_{k=p+1}^{\infty}(\Gamma(k, q) / \gamma(p, q)) b_{k} z^{k-q}} \\
& =z^{m-n}+c_{m-n+1} z^{m-n+1}+\cdots \in \mathscr{T}(m-n),
\end{aligned}
$$

where $\gamma(k, q)$ and $\Gamma(p, q)$ are defined by (10) and (11), respectively. Define $v(z)$ by

$$
\frac{\gamma(m, q)}{\gamma(n, q)} \cdot \frac{f^{(q)}(z)}{g^{(q)}(z)}=v(z) .
$$

Clearly, it is easily seen that $v(z)$ satisfies the conditions of Lemma 1 . The definition in (21) clearly gives us

$$
\begin{aligned}
& \frac{\gamma(m, q)}{\gamma(n, q)} \cdot \frac{f^{(1+q)}(z)}{g^{(1+q)}(z)} \\
& \quad=v(z)\left(1+\frac{z v^{\prime}(z)}{v(z)} \cdot \frac{g^{(q)}(z)}{z g^{(1+q)}(z)}\right) .
\end{aligned}
$$

Assume now that there exists a point $z_{0} \in \mathbb{U}$ such that

$$
\max _{|z| \leq\left|z_{0}\right|}|w(z)|=\left|w\left(z_{0}\right)\right|=1, \quad(z \in \mathbb{U}) .
$$

Then, applying Lemma 1, it follows from (16) and (22) that

$$
\begin{aligned}
& \left|\frac{\gamma(m, q)}{\gamma(n, q)} \cdot \frac{f^{(1+q)}(z)}{g^{(1+q)}(z)}\right|_{z=z_{0}} \mid \\
& \quad=\left|v(z)\left(1+\frac{z v^{\prime}(z)}{v(z)} \cdot \frac{g^{(q)}(z)}{z g^{(1+q)}(z)}\right)\right|_{z=z_{0}} \mid \\
& \quad \geq 1+\Re e\left(\left.\frac{z v^{\prime}(z)}{v(z)} \cdot \frac{g^{(q)}(z)}{z g^{(1+q)}(z)}\right|_{z=z_{0}}\right) \\
& \quad=1+c \cdot \mathfrak{R} e\left(\left.\frac{g^{(q)}(z)}{z g^{(1+q)}(z)}\right|_{z=z_{0}}\right) \geq 1+(m-n) \alpha
\end{aligned}
$$

(since $c \geq m-n)$,

which is contradicting to the assertion (17). Hence, $|v(z)|<1$ for all $z$ in the disk $\mathbb{U}$. Thus, the modulus of the identity (21), that is,

$$
\left|\frac{\gamma(m, q)}{\gamma(n, q)} \cdot \frac{f^{(q)}(z)}{g^{(q)}(z)}\right|=|v(z)|
$$

requires inequality (18). This completes the proof of Theorem 3.

Theorem 4. Let $f(z) \in \mathscr{M}(n), g(z) \in \mathscr{M}(m), q<\min \{n, m\}$, $n \in \mathbb{N}, m \in \mathbb{N}$, and $q \in \mathbb{N}_{0}$.

$$
\begin{aligned}
& \text { If } \\
& \mathfrak{R} e\left\{z\left(\frac{f^{(1+q)}(z)}{f^{(q)}(z)}-\frac{g^{(1+q)}(z)}{g^{(q)}(z)}\right)\right\}<m-n+\frac{1}{2},
\end{aligned}
$$

$(z \in \mathbb{D})$,

then

$$
\mathfrak{R} e\left(z^{n-m} \frac{f^{(q)}(z)}{g^{(q)}(z)}\right)>0, \quad(z \in \mathbb{D}) .
$$

Proof. Let the functions $f(z) \in \mathscr{M}(n)$ and $g(z) \in \mathscr{M}(m)$ be given by (19). Then, in view of Definition (20), define again an analytic function $w(z)$ by

$$
\begin{aligned}
& \frac{\gamma(m, q)}{\gamma(n, q)} \cdot \frac{f^{(q)}(z)}{g^{(q)}(z)} \\
& \quad=z^{m-n}\left[1+c_{1} z+c_{2} z^{2}+c_{3} z^{3}+\cdots\right] \\
& \quad=z^{m-n}[1+w(z)] .
\end{aligned}
$$

It is obvious that $w(z)$ is analytic in $\mathbb{U}$ with $w(0)=0$. By differentiating $w(z)$ in (28) logarithmically, we find that

$$
z\left(\frac{f^{(1+q)}(z)}{f^{(q)}(z)}-\frac{g^{(1+q)}(z)}{g^{(q)}(z)}\right)=m-n+\frac{z w^{\prime}(z)}{1+w(z)},
$$

$(z \in \mathbb{D})$,

and also suppose that there exists a point $z_{0} \in \mathbb{U}$ such that

$$
\max _{|z| \leq\left|z_{0}\right|}|w(z)|=\left|w\left(z_{0}\right)\right|=1, \quad\left(w\left(z_{0}\right)=e^{i \theta} \neq-1\right) .
$$

Then, applying Lemma 1, it follows from (29) that

$$
\begin{aligned}
\mathfrak{R} e & {\left[\left.z\left(\frac{f^{(1+q)}(z)}{f^{(q)}(z)}-\frac{g^{(1+q)}(z)}{g^{(q)}(z)}\right)\right|_{z=z_{0}}\right] } \\
& =m-n+c \cdot \mathfrak{R}\left(\frac{e^{i \theta}}{1+e^{i \theta}}\right) \geq m-n+\frac{1}{2}
\end{aligned}
$$

(since $c \geq 1$ ),

which contradicts the assumption given by (26). Thus, for all $z \in \mathbb{U},|v(z)|<1$ holds. So, (28) immediately yields inequality (27). Therefore, the desired proof is completed.

Theorem 5. Let $f(z) \in M(p), p \geq q$, and $z \in \mathbb{D}$, and also let the following inequality:

$$
\mathfrak{R} e\left(p+\frac{z f^{(1+q)}(z)}{f^{(q)}(z)}+q\right) \begin{cases}<(2 \delta)^{-1}, & \text { when } \delta>0 \\ >(2 \delta)^{-1}, & \text { when } \delta<0\end{cases}
$$


hold. Then

$$
\mathfrak{R} e\left\{\left(\frac{z^{p+q} f^{(q)}(z)}{\gamma(p, q)}\right)^{\delta}\right\}>0, \quad(\delta \neq 0),
$$

where the value of the above complex power is taken to be as its principal value and $\gamma(p, q)$ is given by $(10)$.

Proof. Let $f(z) \in \mathscr{M}(p)$. In view of (9), we again define a function $w(z)$ by

$$
\left(\frac{z^{p+q} f^{(q)}(z)}{\gamma(p, q)}\right)^{\delta}=1+w(z)
$$

we can easily see that $w(0)=0$ and also $w(z)$ is analytic in $\mathbb{U}$. By differentiating logarithmically (34), we receive

$$
\begin{aligned}
\left(p+\frac{z f^{(1+q)}(z)}{f^{(q)}(z)}+q\right)=\frac{1}{\delta} \cdot \frac{z w^{\prime}(z)}{1+w(z)} & \\
& (z \in \mathbb{U} ; w(z) \neq \equiv-1) .
\end{aligned}
$$

Now assuming that there exists a point $z_{0} \in \mathbb{U}$ such that $\max \left\{|w(z)|:|z| \leq\left|z_{0}\right|\right\}=\left|w\left(z_{0}\right)\right|=1$, by using Lemma 1 , we then have $z_{0} w^{\prime}\left(z_{0}\right)=c w\left(z_{0}\right)(c \geq n \geq 1)$ and also take $w\left(z_{0}\right)=e^{i \theta} \neq-1$. Thus, with the help of (35), it can be determined that

$$
\begin{aligned}
& \mathfrak{R} e\left\{\left.\left(p+\frac{z f^{(1+q)}(z)}{f^{(q)}(z)}+q\right)\right|_{z=z_{0}}\right\}=\frac{1}{\delta} \\
& \cdot \mathfrak{R} e\left(\left.\frac{z w^{\prime}(z)}{1+w(z)}\right|_{z=z_{0}}\right) \begin{cases}\geq(2 \delta)^{-1}, & \text { when } \delta>0, \\
\leq(2 \delta)^{-1}, & \text { when } \delta<0,\end{cases}
\end{aligned}
$$

which contradict the cases of (32), respectively. Hence, we conclude that $|w(z)|<1$ for all $z \in \mathbb{U}$, and Definition (34) immediately yields inequality (33). This completes the related proof.

This paper includes several useful results which will be important and/or interesting for analytic and geometric function theory $[1,2]$. We want to point only two special results of the main results out. The others are here omitted.

Remark 6. If we take $\delta=1$ in Theorem 5 , then Theorem 5 requires Theorem 2 .

If we take $q=0$ in Theorem 5 , then we receive the following corollary.

Corollary 7. Let $f(z) \in \mathscr{M}(p), \delta \neq 0$, and $z \in \mathbb{D}$. Then

$$
\begin{gathered}
p+\mathfrak{R e}\left(\frac{z f^{\prime}(z)}{f(z)}\right) \begin{cases}<\frac{1}{2 \delta}, & \text { if } \delta>0 \\
>\frac{1}{2 \delta}, & \text { if } \delta<0\end{cases} \\
\Longrightarrow \mathfrak{R e}\left\{\left[z^{p} f(z)\right]^{\delta}\right\}>0,
\end{gathered}
$$

or, equivalently,

$$
\begin{aligned}
& \mathfrak{R} e\left\{\left[z^{p} f(z)\right]^{\delta}\right\} \leq 0 \\
& \Longrightarrow p+\mathfrak{R} e\left(\frac{z f^{\prime}(z)}{f(z)}\right) \begin{cases}\geq \frac{1}{2 \delta}, & \text { if } \delta>0 \\
\leq \frac{1}{2 \delta}, & \text { if } \delta<0,\end{cases}
\end{aligned}
$$

where the value of the above complex power is considered to be its principal value.

\section{Conflict of Interests}

The authors declare that there is no conflict of interests.

\section{References}

[1] P. L. Duren, Univalent Functions, vol. 259 of Grundlehren der Mathematischen Wissenschaften, Springer, New York, NY, USA, 1983.

[2] A. W. Goodman, Univalent Functions. Volumes I and II, Polygonal Publishing House, Washington, NJ, USA, 1983.

[3] H. Irmak, G. Tinaztepe, N. Tuneski, and M. San, "An ordinary differential operator and its applications to certain classes of multivalently meromorphic functions," Bulletin of Mathematical Analysis and Applications, vol. 1, no. 2, pp. 17-22, 2009.

[4] H. Irmak and N. E. Cho, "A differential operator and its applications to certain multivalently analytic functions," Hacettepe Journal of Mathematics and Statistics, vol. 36, no. 1, pp. 1-6, 2007.

[5] N. E. Cho, "Inclusion properties for certain classes of meromorphic functions associated with a family of linear operators," Journal of Inequalities and Applications, no. 1, Article ID 1147069, 2009.

[6] E. Entisar and M. Darus, "Some properties of differential operator associated with generalized hypergeometric functions," Tamkang Journal of Mathematics, vol. 46, no. 1, pp. 75-83, 2015.

[7] T. N. Shanmugam, S. Sivasubramanian, and S. Owa, "On sandwich results for some subclasses of analytic functions involving certain linear operator," Integral Transforms and Special Functions, vol. 21, no. 1, pp. 1-11, 2010.

[8] H. Irmak and R. K. Raina, "Certain properties arising from some inequalities concerning subclasses of multivalently analytic functions," Mathematical Inequalities and Applications, vol. 10, no. 2, pp. 327-334, 2007.

[9] Y. Shimoda, N. Uyanik, and S. Owa, "Notes on radius properties of $p$-valently starlike functions," Arabian Journal for Science and Engineering, vol. 36, no. 8, pp. 1635-1640, 2011.

[10] I. S. Jack, "Functions starlike and convex of order," Journal of the London Mathematical Society, vol. 3, pp. 469-474, 1971.

[11] S. S. Miller and P. T. Mocanu, Differential Subordinations, Theory and Applications, Marcel Dekker, New York, NY, USA, 2000. 


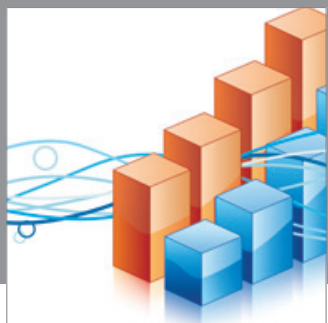

Advances in

Operations Research

mansans

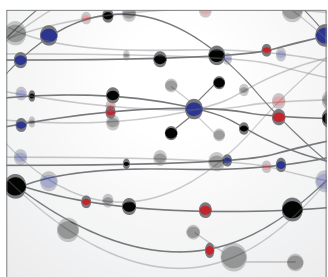

The Scientific World Journal
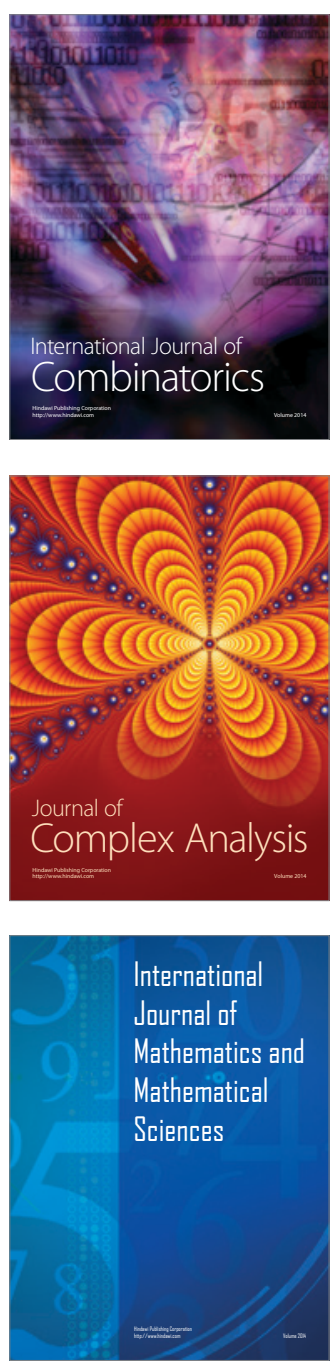
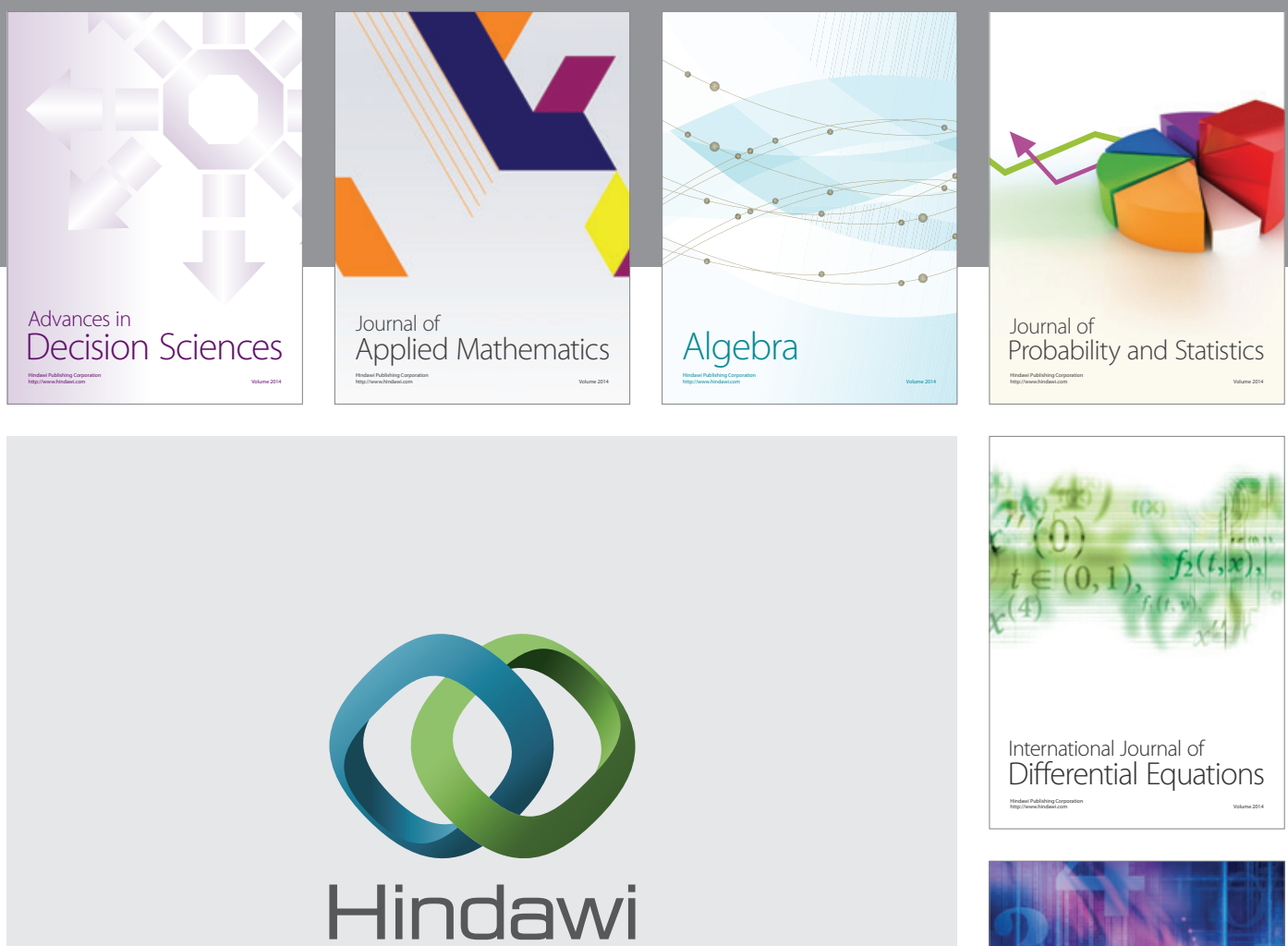

Submit your manuscripts at http://www.hindawi.com
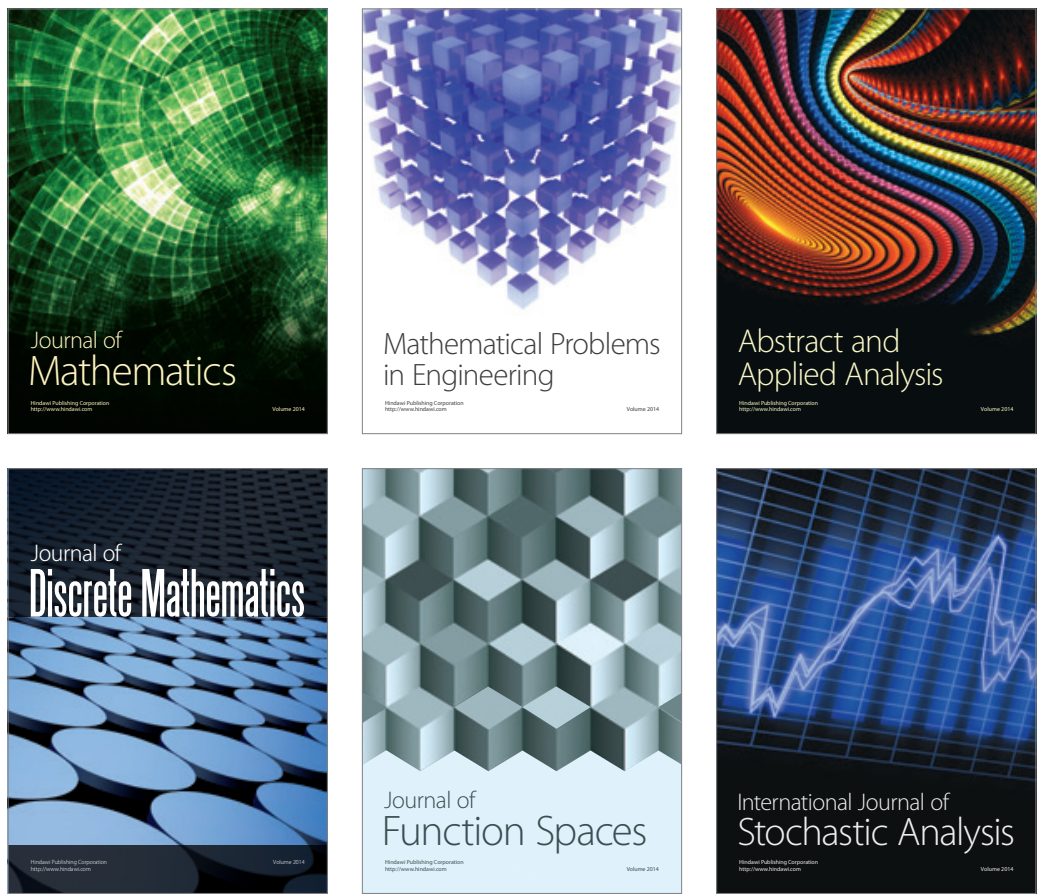

Journal of

Function Spaces

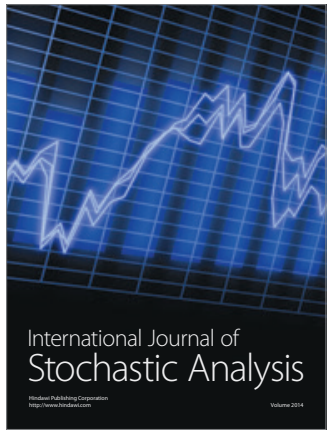

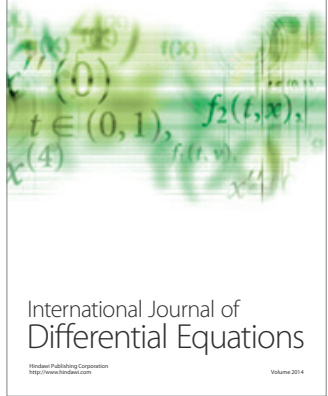
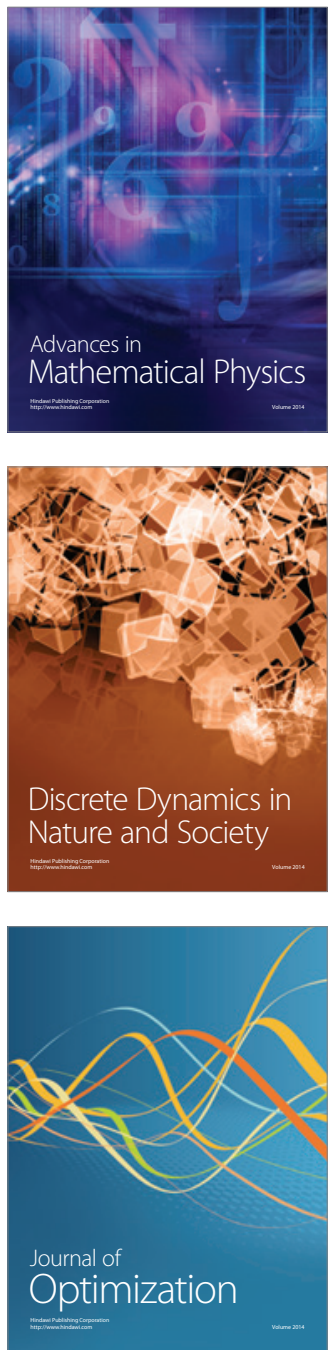